\title{
Article
}

\section{Validation of the simplified modified Rankin scale for stroke trials: Experience from the ENCHANTED alteplase-dose arm}

Chen, Xiaoying, Li, Jingwei, Anderson, Craig S, Lindley, Richard I, Hackett, Maree, Robinson, Thompson, Lavados, Pablo M, Wang, Xia, Arima, Hisatomi, Chalmers, John and Delcourt, Candice

Available at http://clok.uclan.ac.uk/31527/

Chen, Xiaoying, Li, Jingwei, Anderson, Craig S, Lindley, Richard I, Hackett, Maree ORCID: 0000-0003-1211-9087, Robinson, Thompson, Lavados, Pablo M, Wang, Xia, Arima, Hisatomi et al (2021) Validation of the simplified modified Rankin scale for stroke trials: Experience from the ENCHANTED alteplase-dose arm. International Journal of Stroke, 16 (2). pp. 222-228. ISSN 1747-4930

It is advisable to refer to the publisher's version if you intend to cite from the work. http://dx.doi.org/10.1177/1747493019897858

For more information about UCLan's research in this area go to

http://www.uclan.ac.uk/researchgroups/ and search for <name of research Group>.

For information about Research generally at UCLan please go to http://www.uclan.ac.uk/research/

All outputs in CLoK are protected by Intellectual Property Rights law, including Copyright law. Copyright, IPR and Moral Rights for the works on this site are retained by the individual authors and/or other copyright owners. Terms and conditions for use of this material are defined in the policies page. 


\section{International}

\section{A MeoLine listeo, peer revieweo jouranal}

FOR STROKE PRACTITIONERS AROUID THE WORLO
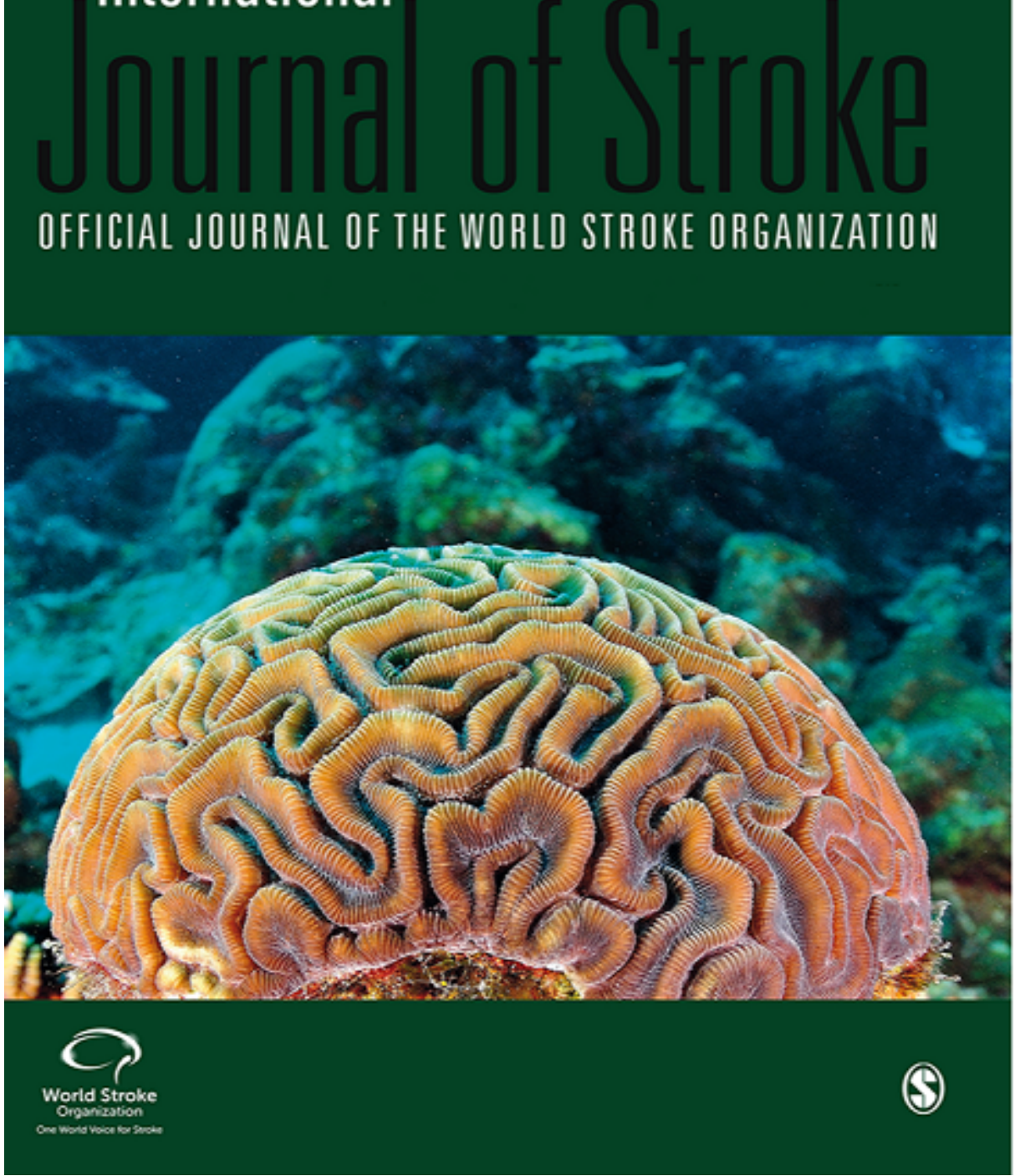

Validation of the simplified modified Rankin scale for stroke trials: experience from the ENCHANTED alteplase-dose arm

\begin{tabular}{|c|c|}
\hline Journal: & International Journal of Stroke \\
\hline Manuscript ID & IJS-07-19-7266.R2 \\
\hline Manuscript Type: & Research \\
\hline $\begin{array}{r}\text { Date Submitted by the } \\
\text { Author: }\end{array}$ & 24-Oct-2019 \\
\hline omplete List of Authors: & Chen, Xiaoying; The George Institute for Global Health, \\
\hline
\end{tabular}




\begin{tabular}{|c|c|}
\hline & $\begin{array}{l}\text { li, jINGWEI; George Institute for Global Health, } \\
\text { Anderson, Craig; University of New South Wales, The George Institute } \\
\text { for Global Health, Faculty of Medicine; Peking University Health Sciences } \\
\text { Centre, ; Royal Prince Alfred Hospital } \\
\text { Lindley, Richard; The University of Sydney Westmead Clinical School } \\
\text { Hackett, Maree; The George Institute for Global Health, Neurological \& } \\
\text { Mental Health Division, The University of Sydney; The University of } \\
\text { Central Lancashire, School of Health } \\
\text { Robinson, Tom } \\
\text { Lavados, Pablo; Universidad del Desarrollo, Departamento de Neurologia } \\
\text { y Psiquiatria } \\
\text { Wang, Xia; The George Institute for Global Health, Neurological \& } \\
\text { Mental Health Division } \\
\text { Arima, Hisatomia; The George Institute for Global Health, NMH; Fukuoka } \\
\text { University, Department of Preventive Medicine and Public Health, Faculty } \\
\text { of Medicine } \\
\text { Chalmers, John } \\
\text { Delcourt, Candice; The George Institute, }\end{array}$ \\
\hline Keywords: & $\begin{array}{l}\text { simplified modified Rankin scale questionnaire, modified Rankin scale, } \\
\text { ischemic stroke, Clinical trial, health outcome, Thrombolysis }\end{array}$ \\
\hline
\end{tabular}


Validation of the simplified modified Rankin scale for stroke trials: experience from the ENCHANTED alteplase-dose arm

Xiaoying Chen BPharm BMgt, ${ }^{1,2}$ Jingwei Li MD PhD, ${ }^{1,3,4}$ Craig S. Anderson MD PhD, $, 15,6$

Richard I. Lindley MD, ${ }^{7}$ Maree L Hackett PhD, ${ }^{1,2,8}$ Thompson Robinson MD, ${ }^{9,10}$ Pablo M. Lavados MD MPH, ${ }^{11,12}$ Xia Wang PhD,${ }^{1}$ Hisatomi Arima PhD, ${ }^{1,13}$ John Chalmers MD PhD, ${ }^{1}$ Candice Delcourt MD PhD, ${ }^{1,2,6}$ for the ENCHANTED Investigators*

${ }^{1}$ The George Institute for Global Health, Faculty of Medicine, University of New South Wales, Sydney, Australia.

${ }^{2}$ Faculty of Medicine and Health, University of Sydney, NSW, Australia

${ }^{3}$ Department of Cardiology, People's Liberation Army General Hospital, Beijing, China

${ }^{4}$ Department of Cardiology, Xinqiao Hospital, Third Military Medical University, Chongqing, China

${ }^{5}$ The George Institute China at Peking University Health Science Center, Beijing, PR China

${ }^{6}$ Neurology Department, Royal Prince Alfred Hospital, Sydney Health Partners, Sydney, NSW, Australia

${ }^{7}$ Westmead Clinical School, University of Sydney, NSW, Australia;

${ }^{8}$ Faculty of Health and Wellbeing, University of Central Lancashire, UK

${ }^{9}$ Department of Cardiovascular Sciences, University of Leicester, Leicester, UK

${ }^{10}$ NIHR Biomedical Research Centre, Leicester, UK

${ }^{11}$ Departamento de Ciencias Neurológicas, Facultad de Medicina, Universidad de Chile, Santiago, Chile

${ }^{12}$ Unidad de Neurología Vascular, Servicio de Neurología, Departamento de Neurología y Psiquiatría, Clínica Alemana de Santiago, Facultad de Medicina, Universidad del Desarrollo, Santiago, Chile

${ }^{13}$ Department of Preventive Medicine and Public Health, Faculty of Medicine, Fukuoka University, Fukuoka, Japan

Correspondence to:

Professor Craig Anderson, The George Institute for Global Health, University of New South Wales PO Box M201, Missenden Road, NSW 2050, Sydney, Australia

E: canderson@georgeinstitute.org.au; T: +61 29993 4521; F : +61 299934502

Word Count: abstract 265; body 2398

Key words: simplified modified Rankin scale questionnaire, modified Rankin scale, ischemic stroke, clinical trial, health outcome

Cover title: Validity of simplified mRS questionnaire

Supp. Tables: 3

Figures: 2 
Subject Terms: quality and outcomes, ischemic stroke List of tables and figures:

Supp. Table 1. Correlation between smRSq and mRS scores at Day 90

Supp. Table 2. Independent predictors of smRSq and mRS at Day 90

Supp. Table 3. Comparison of treatment effects using $\mathrm{mRS}$ and smRSq in the alteplasedose arm of the ENCHANTED trial

Figure 1. Bubble plot of agreement between smRSq and mRS at Day 90

Figure 2. ROC curves for predictive models of mRS and smRSq at Day 90. 


\section{Abstract}

2 Background and Aims: The structured, simplified modified Rankin scale questionnaire

3 (smRSq) may increase reliability over the interrogative approach to scoring the modified

4 Rankin scale (mRS) in acute stroke research and practice. During the conduct of the alteplase-

5 dose-arm of the international ENhanced Control of Hypertension ANd Thrombolysis StrokE

6 stuDy (ENCHANTED), we had an opportunity to compare each of these approaches to

7 outcome measurement.

8 Methods: Baseline demographic data were recorded together with the National Institutes of

9 Health Stroke Scale (NIHSS). Follow-up measures obtained at 90 days included mRS, smRSq, 10 and the 5-Dimension European Quality of life scale (EQ-5D). Agreements between smRSq 11 and mRS were assessed with the Kappa statistic. Multiple logistic regression was used to identify baseline predictors of Day $90 \mathrm{smRSq}$ and mRS scores. Treatment effects, based on Day $90 \mathrm{smRSq} / \mathrm{mRS}$ scores were tested in logistic and ordinal logistic regression models.

Results: SmRSq and mRS scores had good agreement (weighted Kappa 0.79, 95\% confidence interval [CI] 0.78-0.81), whilst variables of age, atrial fibrillation, diabetes mellitus, pre-morbid mRS (1 vs. 0), baseline NIHSS scores and imaging signs of cerebral ischemia, similarly predicted their scores. Odds ratios for death or disability, and ordinal shift, 90 day mRS scores using smRSq were 1.05 (95\% CI 0.91-1.20; one-sided $p=0.23$ for noninferiority) and 0.98 (95\% CI $0.87-1.11 ; \mathrm{P}=0.02$ for noninferiority), similar to those using $\mathrm{mRS}$.

Conclusions: This study demonstrates the utility of the smRSq in a large, ethnically diverse clinical trial population. Scoring of the smRSq shows adequate agreement with the standard $\mathrm{mRS}$, thus confirming it is a reliable, valid and useful alternative measure of functional status after acute ischemic stroke.

Clinical Trial registration URL: http://www.clinicaltrials.gov. Unique identifier: 


\section{Introduction and Aims}

27

The modified Rankin scale (mRS) is the most popular assessment tool for measuring overall functional status in patients who have suffered a stroke or other form of neurological disability, ${ }^{1}$ both in clinical practice and research. ${ }^{2,3}$ However, due to criticism being raised over subjectivity in aspects of its categorization/scoring, ${ }^{4}$ Bruno et al. developed the short, structured, simplified modified Rankin scale questionnaire $(\mathrm{smRSq})^{5,6}$ which has been shown to correlate with the size of the ischemic lesion, ${ }^{6}$ health-related quality of life, ${ }^{7}$ and neurological severity ${ }^{8}$ in small single center studies. The smRSq has also shown good reliability and validity in Chinese stroke patients. ${ }^{9}$ However, it has not been validated in a broader population or in the context of international research where the mRS remains the gold standard method of outcome assessment. We aimed to compare scores on the mRS and smRSq, their predictor variables, their correlation with neurological impairment on the National Institutes of Health Stroke Scale (NIHSS) and health-related quality of life on the 5Dimension European Quality of life scale (EQ-5D), and treatment effects using them as outcome measures, among participants of the alteplase-dose arm of the Enhanced Control of Hypertension and Thrombolysis Stroke study (ENCHANTED).

\section{Methods}

\section{Study design}

ENCHANTED was an international, multicenter, quasi-factorial, prospective, randomized, open, blinded outcome assessed, clinical trial that assessed the effectiveness of low versus standard dose intravenous alteplase, and intensive versus guideline-recommended blood pressure (BP) management, in thrombolysis-eligible patients with acute ischemic stroke, the details of which are described elsewhere. ${ }^{10,11}$ In brief, the first arm of the trial assessed 0.6 $\mathrm{mg} / \mathrm{kg}$ compared to $0.9 \mathrm{mg} / \mathrm{kg}$ alteplase in 3310 patients (age $\geq 18$ years) within 4.5 hours of 
the onset of symptoms and followed up these patients to 90 days. The primary endpoint was death or disability defined by scores of 2 to 6 on the mRS. The trial was approved by local ethics committees and regulatory bodies, and written informed consent was obtained from the patient or an appropriate surrogate. The trial is registered at ClinicalTrials.gov (NCT01422616).

\section{Measures}

Demographics, clinical characteristics including the severity of neurological impairment on the NIHSS, were recorded in participants at the time of enrolment (baseline). The trial excluded patients with pre-morbid functional impairment (mRS scores $>1)$ but collected estimated premorbid mRS ( 0 or 1$)$ for those included. Signs of cerebral ischemia on brain imaging, and any evidence of proximal vessel occlusion on computed tomographic angiography (CTA) or magnetic resonance angiography (MRA), were reported by clinicians. Assessors with a health professional background (doctors, nurses or scientists) blind to treatment allocation and who had received in-person and online training (https://secure.trainingcampus.net), recorded mRS and smRSq scores by telephone or face-to-face interview in patients or a suitable proxy at 28 and 90 days post-randomisation. These outcome assessors had no mandatory training in the use of smRSq. They were advised to first assess patients with the mRS and then immediately administer the smRSq, as listed on the case report form. The 7-item mRS covers no symptoms (score 0), symptoms but no significant disability (1), slight disability (2), moderate disability (3), moderately severe disability (4), severe disability (5), and death (6). The smRSq takes on average 1.7 minutes to administer, ${ }^{7}$ and represents $\mathrm{mRS}$ items through yes/no answers to 5 questions addressing key functional states: living alone without any help from another person for bathing, toileting, shopping, preparing or getting meals, and managing finances; doing everything as before the stroke; being back to pre-stroke status; walking without help from another person; and being bedridden or needing constant supervision. The EQ-5D, which was 
also administered directly in a patient or proxy at 28 and 90 days, defines the state of general health across five dimensions (mobility, self-care, usual activities, pain/discomfort, and anxiety/depression) with three levels of responses within each dimension (no problems, some/moderate problems, and severe problems). The EQ-5D utility score integrates the ratings of the 5 dimensions into a single score, calculated using population-based preference weights for each subscale. The weights used in the present analyses were derived from a study based on a representative sample of the UK population. ${ }^{12}$ Utility scores express HRQoL quantitatively as a fraction of perfect health, with a score of 1 representing perfect health, a score of 0 representing death, and negative scores (minimum score -0.594) representing health states considered worse than death. ${ }^{13}$

\section{Statistical analysis}

Strength of agreement on ordinal analysis ${ }^{14}$ of the smRSq and mRS at Day 90 were assessed through Cohen's unweighted kappa $(\mathrm{K})$ values of $\leq 0$ (poor), 0.01-0.20 (slight), 0.21-0.40 (fair), 0.41-0.60 (moderate), 0.61-0.80 (substantial), and 0.81-1 (almost perfect), and weighted kappa $(\mathrm{Kw})$ values of $\leq 0.20$ (poor), $0.21-0.40$ (fair), $0.41-0.60$ (moderate), $0.61-0.80$ (good), and 0.81-1.00 (very good) agreement. ${ }^{15}$ Multiple logistic regression was used to build prediction models for scores on the mRS and smRSq at Day 90, and to calculate C-indexes. Significant predictors $(\mathrm{P}<0.05)$ from the univariate analyses were tested in multiple logistic regression models for their associations with outcomes. The non-significant covariates were removed until all the remaining predictors were statistically significant $(\mathrm{P}<0.05)$. Collinearity between variables were checked. Baseline variables included in the models were: age ( $<65$ vs. $\geq 65$ years), sex, estimated prestroke function on mRS ( 0 vs. 1$)$, baseline NIHSS score, history of atrial fibrillation (AF), diabetes mellitus, hypertension, previous stroke, coronary artery disease, and hypercholesterolemia, use of aspirin/other antiplatelet agent(s), and warfarin/other anticoagulation, and visible early ischemic change and proximal vessel occlusion on imaging. 
100 Correlations between smRSq and mRS at Day 90, and with NIHSS and EQ-5D utility scores

101

102

103

104

105

106

107

108

109

110

111

112

113

114

115

116

117

118 at Day 90, were analyzed using Spearman correlation, with the $\mathrm{r}$ coefficient graded as $0.2-0.4$ (weak), 0.4-0.7 (moderate), and 0.7-1.0 (strong). The treatment effects comparing low-dose alteplase to standard-dose alteplase in the trial were tested using scores derived from smRSq, to compare with the study results generated using $\mathrm{mRS}$. The noninferiority margin was $1.14,{ }^{10,11}$ that is for the upper boundary of the $95 \%$ confidence interval (CI) for the odds ratio (OR) with low-dose alteplase as compared with standard-dose alteplase, of less than 1.14. Single logistic regression was used to test and estimate unadjusted OR of death and disability (mRS 2 to 6). Multiple logistic regression were used for adjusted OR in intention to treat and per protocol populations. For shift analyses of the smRSq scores, ordinal logistic regression was used. The variables adjusted in treatment effect analyses include site, time from symptom onset to randomisation, score as a continuous measure on the NIHSS, age, sex, ethnicity, premorbid mRS score ( 0 or 1$)$, pre-morbid use of aspirin, other antiplatelet agent or warfarin, and any history of stroke, coronary artery disease, diabetes mellitus and atrial fibrillation (AF). Testing was undertaken for the degree of agreement between smRSq and mRS at Day 28 using Kappa $(\mathrm{K})$ and weighted Kappa $(\mathrm{Kw})$, and for the strength of correlations between smRSq or mRS at Day 28, and NIHSS or EQ-5D utility scores at Day 28, using Spearman correlation with the $\mathrm{r}$ coefficient (Supplementary Appendix). P values $<0.05$ were regarded as significant. SAS enterprise 7.1 was used in all analyses.

\section{Data sharing}

The authors confirm that the data supporting the findings of this study are available within the article and/or its supplementary materials. Individual participant data used in these analyses can be shared by request from any qualified investigators via the Research Office of The George Institute for Global Health, Australia.

\section{Results}


There were 3204 acute ischemic stroke patients with NIHSS scores recorded at baseline, and mRS, smRSq and EQ-5D scores recorded at Day 90. Agreement between smRSq and mRS scores occurred in 2051 (64\%) patients (Supp Table 1, Figure 1), and overall was moderategood (K 0.57, 95\% CI 0.55-0.59, and Kw 0.79, 95\% CI 0.78-0.81).

Supplementary Table 2 shows the variables remained in the prediction models were common to both the smRSq and mRS at Day 90 after successively removing non-significant covariates; these included age ( $>65$ years), AF, diabetes mellitus, pre-morbid symptoms, NIHSS scores and signs of cerebral ischemia on imaging. C-indexes for the model fit were similar for the smRSq and mRS $(0.74,95 \%$ CI $0.72-0.76$, and $0.75,95 \%$ CI $0.73-0.77$, mRS, respectively) (Figure 2).

Concordance was also evident for baseline NIHSS scores (positive correlation; r 0.442, $\mathrm{P}<0.0001$ and $\mathrm{r} 0.455, \mathrm{P}<0.0001$, respectively) and EQ-5D utility score (negative correlation; $\mathrm{r}-0.836, \mathrm{P}<0.0001$, and $\mathrm{r}-0.874, \mathrm{P}<0.0001$, respectively) and smRSq and mRS at Day 90.

Comparisons of the treatment effects using smRSq and mRS are presented in Supp. Table 3. Both the dichotomous and ordinal outcomes using smRSq were similar to the outcomes from mRS. The unadjusted dichotomous outcome (score of smRSq 2 to 6), which was used to compare with the primary outcome of the alteplase-dose arm of the trial (OR 1.09, 95\% CI 0.95-1.25; one sided $\mathrm{P}=0.51$ for noninferiority), occurred in 886 of 1609 patients $(55.1 \%)$ in the low-dose group and in 863 of 1600 patients (53.9\%) in the standard-dose group (OR 1.05, 95\% CI $0.91-1.20$; one-sided $\mathrm{P}=0.23$ for noninferiority). In the unadjusted shift analysis on smRSq scores comparing low-dose alteplase to standard-dose alteplase, the OR was 0.98 (95\% CI $0.87-1.11 ; \mathrm{P}=0.02$ for noninferiority) similar to that for mRS shift scores (OR 1.0; 95\% CI 0.89-1.13; $\mathrm{P}=0.04$ for noninferiority).

The results for agreement between smRSq and mRS at Day 28, and correlations with NIHSS 
and EQ-5D utility score at Day 28, are included in the supplementary appendix.

150

151

152

153

154

155

156

157

158

159

160

161

162

163

164

165

166

167

168

169

170

171

172

\section{Discussion}

Our study validates the smRSq as a suitable stroke outcome measure by showing comparable scoring to the conventional mRS, similar level of moderate-strong correlations with the NIHSS and EQ-5D, common predictor variables and similar treatment effects when used as trial outcome.

Dennis et al. $^{3}$ showed similar agreement between the mRS and smRSq using postal or telephone assessment in 225 participants, whilst Yuan et al. ${ }^{9}$ found a higher degree of overall agreement than we have shown in their study of 150 Chinese patients. The factors identified in our predictive models for the smRSq and mRS support other outcome studies. ${ }^{16,17}$ For example, in a multivariable analysis by Wahlgren et al., ${ }^{16}$ older age, high blood glucose, high NIHSS, and infarction on brain imaging were found to predict poor outcome (mortality or dependency) in patients treated with intravenous alteplase, whilst pre-stroke disability was only associated with mortality. Baseline severity, history of diabetes mellitus, ischemic stroke, and peripheral artery disease have also been reported to predict recovery after disabling ischemic stroke. ${ }^{17}$ Katzan et al. ${ }^{18}$ showed only a moderate correlation $(r=-0.53, p<0.01)$ between the $\mathrm{mRS}$ and EQ-5D utility score, possibly due to the greater number of patients with $\mathrm{mRS}$ scores of $0-2(75 \%)$, which has shown a lower correlation with EQ-5D ${ }^{19}$, than in the ENCHANTED ${ }^{10}$ ( $65 \%)$. Another study showed the smRSq had moderate correlation with the physical $(\mathrm{r}=0.50$, $\mathrm{P}=0.005)$ but only slight correlation with the mental components $(\mathrm{r}=0.36, \mathrm{P}=0.048)$ of the 12 item short form questionnaire. ${ }^{5}$

More severe strokes (NIHSS scores $>10$ ) are associated with higher mRS scores at hospital discharge. ${ }^{20}$ NIHSS scores at Day 2 are a good predictor of mRS scores $>3$ at 90 days. ${ }^{21}$ In a study of acute ischemic stroke patients treated with mechanical thrombectomy, NIHSS scores 
at baseline and hospital discharge were each significantly associated with 90 -day mRS scores. ${ }^{22}$ Another study has shown a similar moderate level of correlation between initial NIHSS and Day 90 smRSq scores $\left(r=0.69, \mathrm{R}^{2}=0.47, \mathrm{P}<0.001\right)^{5}$ to our study.

The smRSq appears easy to administer and automatically calculates a final score from the structured responses to five questions, whereas the mRS often requires the assessor to make a judgment call in deciding which category best fits a certain grading of disability or level of dependency. While training in the use of the mRS is often used to decrease error, this can be resource intensive for large studies. It is interesting to note that a high percentage of patients who scored 1 or 2 on the mRS scored 3 on smRSq in our study. One explanation could be that a high proportion of ENCHANTED patients experienced acalculia and difficulty managing finances without major motor disability after suffering a left middle cerebral artery stroke. This may have resulted in them answering negatively the first question of the smRSq, resulting in a score $\geq 3$. Another explanation is broader cognitive impairment but we did not collect such information in the study.

Our analyses found that similar factors were predictors of smRSq and mRS. This confirms the good correlation between the two scales and re-enforces that they are well-known predictors of poor outcome. Similarly, the correlation between smRSq and mRS is good which is not surprising as both scales correlated similarly with the NIHSS and EQ-5D.

In reviewing the treatment effects of the alteplase-dose arm of ENCHANTED, use of the smRSq similarly failed to show that low-dose alteplase was noninferior to standard-dose alteplase with respect to death or disability at Day 90, but was non-inferior with respect to ordinal shift of smRSq scores, which is consistent with those results using mRS. ${ }^{10}$ This again reflects good correlation between the two measures, and for the smRSq to provide a comparable assessment of a treatment effect to that on the mRS. 
197 Strengths of this study is the large database of prospectively and systematically assessed 198 patients from a variety of countries and ethnic backgrounds. There are some limitations 199 including that these were post-hoc analyses and that the same outcome assessors rated the mRS 200 and smRSq. However, the Day 90 assessment case report form was structured for sequential 201 recording of the $\mathrm{mRS}$ followed by $\mathrm{smRSq}$, and these people were not provided with scoring answers to the smRSq questions. Another issue is that as patients with pre-morbid functional impairment/disability (mRS $>1$ ) were excluded from the trial, we are unable to provide an assessment of any influence of this factor on the correlation between the measures. Moreover, the finding of large proportion of patients in the score of 3 using smRSq, similarly shown in the FOCUS trial, ${ }^{23}$ suggests distribution of patients across categories may differ between mRS and smRSq, which potentially influenced the results of this study. Finally, as this work pertains to a clinical trial involving acute ischemic stroke patients of predominantly mild-moderate severity, caution may be required in generalizing these results to a more severe patient population or in those with acute intracerebral hemorrhage.

211 In summary, our study has shown that the smRSq has comparable scoring and construct to the

212 conventional $\mathrm{mRS}$, and provides a useful, reliable and valid outcome measure in the assessment of patients with acute ischemic stroke. 


\section{Author contributions}

215 XC undertook analyses and wrote the first draft of the manuscript; CD, JL and CSA interpreted 216 the data; other authors provided critical review; all authors contributed to drafting and take 217 responsibility for the content and integrity of this article.

\section{Funding/Support}

219 The ENCHANTED study received grants from the National Health and Medical Research 220 Council (NHMRC) of Australia (Project Grant numbers 1020462 and 1101113), the Stroke Association of the United Kingdom (TSA 2012/01 and 2015/01), the Ministry of Health and the National Council for Scientific and Technological Development of Brazil (CNPQ: 467322/2014-7, 402388/2013-5), the Ministry for Health, Welfare and Family Affairs of the Republic of Korea (HI14C1985). During the completion of this work Maree Hackett was supported by a NHMRC Career Development Fellowship Level 2 (APP1141328) and Craig Anderson a NHMRC Senior Principal Research Fellowship.

\section{Role of the Funders/Sponsors}

The funding bodies had no role in the design and conduct of the analyses and interpretation of the data; and preparation, review, or approval of the manuscript

\section{Conflicts of Interest Disclosures}

Dr. Anderson reports receiving fees for serving on advisory boards from Amgen, Boehringer Ingelheim, and lecture fees and travel support from Takeda. Dr Chalmers reports research grants and lecture fees from Servier for the ADVANCE trial and post-trial follow-up. Dr. Lavados reports receiving fees for serving on the advisory boards from ANGELS initiative and lectures fees from Boehringer Ingelheim and grant support from Bayer and Boehringer Ingelheim. The other authors report no conflicts of interest. 


\section{Acknowledgement}

238 We acknowledge the contribution of the large number of patients, hospital site investigators

239 and coordinators, and central and regional project staff for the ENCHANTED study. We thank

240 EuroQoL Group for providing translations and license for EQ-5D. 


\section{Reference}

242 1. Bamford JM, Sandercock PA, Warlow CP, Slattery J. Interobserver agreement for the assessment of handicap in stroke patients. Stroke 1989;20:828

2. Saver JL, Filip B, Hamilton S, Yanes A, Craig S, Cho M, et al. Improving the reliability of stroke disability grading in clinical trials and clinical practice: the Rankin Focused Assessment (RFA). Stroke 2010;41:992-995

3. Dennis M, Mead G, Doubal F, Graham C. Determining the modified Rankin Score after stroke by postal and telephone questionnaires. Stroke 2012;43:851-853

4. Wilson JT, Hareendran A, Grant M, Baird T, Schulz UG, Muir KW, et al. Improving

5. Bruno A, Shah N, Lin C, Close B, Hess DC, Davis K, et al. Improving modified Rankin Scale assessment with a simplified questionnaire. Stroke 2010;41:1048-1050

6. Bruno A, Shah N, Akinwuntan AE, Close B, Switzer JA. Stroke size correlates with functional outcome on the simplified modified Rankin Scale questionnaire. J Stroke Cerebrovasc Dis 2013;22:781-783

7. Bruno A, Akinwuntan AE, Lin C, Close B, Davis K, Baute V, et al. Simplified modified Rankin Scale questionnaire: reproducibility over the telephone and validation with quality of life. Stroke 2011;42:2276-2279

8. Bruno A, Close B, Switzer JA, Hess DC, Gross H, Nichols III FT, et al. Simplified modified Rankin Scale questionnaire correlates with stroke severity. Stroke $2013 ; 27: 724-727$

9. Yuan JL, Bruno A, Li T, Li SJ, Zhang XD, Li HY, et al. Replication and extension of the simplified modified Rankin Scale in 150 chinese stroke patients. Eur Neurol 
10. Anderson CS, Robinson T, Lindley RI, Arima H, Lavados PM, Lee TH, et al. Lowdose versus standard-dose intravenous alteplase in acute ischemic stroke. $N$ Engl J Med $2016 ; 374: 2313-2323$

11. Huang Y, Sharma VK, Robinson T, Lindley RI, Chen X, Kim JS, et al. Rationale,

12. Dolan P, Gudex C, Kind P, Williams A. A social tariff for EuroQol: results from a UK general population survey. 1995

13. Kind P, Hardman G, Macran S. UK population norms for EQ-5D. 1999

14. Cohen J. Weighted kappa: Nominal scale agreement with provision for scaled disagreement or partial credit. Psychol Bull 1968;70:213-220

15. Brennan P, Silman A. Statistical methods for assessing observer variability in clinical measures. BMJ 1992;304:1491-1494

16. Wahlgren AN, Ahmed GN, Eriksson RN, Aichner OF, Bluhmki OE, Dávalos OA, et al. Multivariable analysis of outcome predictors and adjustment of main outcome results to baseline data profile in randomized controlled trials: Safe Implementation of Thrombolysis in Stroke-Monitoring Study (SITS-MOST). Stroke 2008;39:3316-3322

17. Hankey GJ, Spiesser J, Hakimi Z, Bego G, Carita P, Gabriel S. Rate, degree, and predictors of recovery from disability following ischemic stroke. Neurology 2007;68:1583-1587 
289 18. Katzan IL, Thompson NR, Lapin B, Uchino K. Added value of patient-reported outcome measures in stroke clinical practice. J Am Heart Assoc 2017;6

291

19. Rangaraju S, Haussen D, Nogueira RG, Nahab F, Frankel M. Comparison of 3-month stroke disability and quality of life across modified Rankin Scale categories. Interv Neurol 2017;6:36-41

20. Mihindu E, Mohammed A, Smith T, Brinster C, Sternbergh WC, 3rd, Bazan HA. Patients with moderate to severe strokes (NIHSS score $>10$ ) undergoing urgent carotid interventions within 48 hours have worse functional outcomes. J Vasc Surg $2019 ; 69: 1471-1481$

21. De Raedt S, Brouns R, De Smedt A, Aries MJ, Uyttenboogaart M, Luijckx GJ, et al. The sNIHSS-4 predicts outcome in right and left anterior circulation strokes. Clin Neurol Neurosurg 2013;115:729-731

22. Costalat V, Lobotesis K, Machi P, Mourand I, Maldonado I, Heroum C, et al. 302 Prognostic factors related to clinical outcome following thrombectomy in ischemic stroke (RECOST study): 50 patients prospective study. Eur J Radiol 2012;81:40754082

23. Dennis M, Mead G, Forbes J, Graham C, Hackett M, Hankey GJ, et al. Effects of fluoxetine on functional outcomes after acute stroke (FOCUS): A pragmatic, doubleblind, randomised, controlled trial. The Lancet. 2019;393:265-274 
Table 1. Correlation between smRSq and mRS seores at Day 90

\begin{tabular}{|c|c|c|c|c|c|c|c|c|}
\hline mRS & \multicolumn{8}{|c|}{ smRSq } \\
\hline & $\theta$ & 4 & 2 & 3 & 4 & 5 & 6 & Fotal \\
\hline$\theta$ & $704(88.0)$ & $42(5.3)$ & $11(1.3)$ & $42(5.3)$ & $1(0.1)$ & - & - & 800 \\
\hline 4 & $311(42.4)$ & $266(36.3)$ & $56(7.6)$ & $97(13.2)$ & $3(0.4)$ & - & - & 733 \\
\hline$z$ & $38(8.0)$ & $75(15.8)$ & $167(35.2)$ & $174(36.6)$ & $16(3.4)$ & $5(1.1)$ & - & 475 \\
\hline 3 & $6(1.5)$ & $8(2.0)$ & $22(5.6)$ & $285(72.7)$ & $52(13.3)$ & $19(4.6)$ & - & 392 \\
\hline 4 & $5(1.6)$ & $z(0.6)$ & $z(0.6)$ & $36(11.3)$ & $162(50.9)$ & 111 (34.9) & - & 318 \\
\hline 5 & $z(1.1)$ & - & - & $z(1.1)$ & $15(8.5)$ & $157(89.2)$ & - & 176 \\
\hline 6 & - & - & - & - & - & - & $310(100)$ & 310 \\
\hline Fotal & 1066 & 393 & 258 & 636 & 249 & 292 & 310 & $3204^{*}$ \\
\hline
\end{tabular}

mRS denotes modified Rankin Seale, smRSq simplified modified Rankin Seale questionnaire.

Kappa statistic 0.57 (95\% confidence interval [CI] 0.55-0.59) and weighted Kappa statistic 0.79 (95\% CI $0.78-0.81$ )

*3310 patients were randomized into the alteplase-dose arm, of which 13 were excluded; another 93 patients were excluded from these analyses due to missing $\mathrm{mRS}$ or smRSq data. 
Table 2. Independent predictors of smRSq and mRS at Day 90

\begin{tabular}{|c|c|c|c|c|c|c|}
\hline \multirow[b]{2}{*}{ Variable } & \multicolumn{3}{|c|}{$\begin{array}{c}\text { smRSq } \\
(\mathrm{C}=0.740,95 \% \mathrm{CI} 0.723-0.757)\end{array}$} & \multicolumn{3}{|c|}{$\begin{array}{c}\mathrm{mRS} \\
(\mathrm{C}=0.751,95 \% \mathrm{CI} 0.734-0.767)\end{array}$} \\
\hline & OR & $95 \% \mathrm{CI}$ & p Value & OR & $95 \% \mathrm{CI}$ & p Value \\
\hline Age $>65$ & 1.47 & $1.26-1.71$ & $\leq 0.0001$ & 1.33 & $1.13-1.56$ & 0.0005 \\
\hline Atrial fibrillation & 1.43 & $1.16-1.77$ & 0.0009 & 1.29 & $1.04-1.59$ & 0.019 \\
\hline Diabetes mellitus & 1.25 & $1.03-1.51$ & 0.0245 & 1.37 & $1.13-1.66$ & 0.002 \\
\hline Pre-stroke grade of physical function* & 2.21 & $1.79-2.72$ & $\leqslant 0.0001$ & 2.24 & 1.822 .77 & $\leq 0.0001$ \\
\hline NHHSS & 1.14 & $1.12-1.16$ & $\leqslant 0.0001$ & 1.16 & $1.14-1.17$ & $\leq 0.0001$ \\
\hline Signs of cerebral ischemia on imaging & 1.56 & $1.30-1.88$ & $<0.0001$ & 1.42 & $1.18-1.71$ & 0.0002 \\
\hline
\end{tabular}

C denotes Concordance Index, CI confidence interval, mRS modified Rankin Scale, NHSS National Institutes of Health Stroke Seale, OR odds ratio, smRSq simplified modified Rankin Scale questionnaire

*pre-morbid estimated level of physical function with symptoms, based on a score of 1 on the $\mathrm{mRS}$; the comparison was 1 vs. $\theta$

Significant predictors $(P<0.05)$ from the univariate analyses which were tested in multiple logistic regression models were: sex, history of hypertension, previous stroke, coronary artery disease, hypercholesterolemia, use of aspirin/other antiplatelet agent(s), use of warfarin/other anticoagulation and proximal vessel occlusion. Significance level of stay in the models was $\mathrm{P}<0.05$. 
Table 3. Comparison of treatment effects using mRS and smRSq in the alteplase-dose arm of the ENCHANTED trial

\begin{tabular}{|c|c|c|c|c|c|c|}
\hline \multirow[b]{2}{*}{ Outcome } & \multicolumn{3}{|c|}{ smRSq } & \multicolumn{3}{|c|}{$\mathrm{mRS}$} \\
\hline & OR & $95 \% \mathrm{CI}$ & P value* & OR & $95 \% \mathrm{CI}$ & $\mathrm{P}_{\text {value }}$ \\
\hline \multicolumn{7}{|l|}{ Death or disability: scores 2 to 6 市 } \\
\hline Unadjusted & 1.05 & $0.91-1.2 \theta$ & $\theta .23$ & 1.09 & $0.95-1.25$ & $\theta .51$ \\
\hline Adjusted & 1.06 & $0.91-1.23$ & $\theta .34$ & 1.13 & $0.97-1.31$ & $\theta .88$ \\
\hline Adjusted in per protocol populationf & 1.05 & $\theta .89-1.23$ & $\theta .3 \theta$ & 1.13 & 0.961 .32 & $\theta .89$ \\
\hline \multicolumn{7}{|l|}{ Shift analyses of scores $\theta$ to $6 *$} \\
\hline Unadjusted & 0.98 & $0.87-1.11$ & $\theta .02$ & 1.00 & $0.89-1.13$ & $\theta .04$ \\
\hline Adjusted + & 0.97 & $0.85-1.10$ & $\theta .01$ & 0.99 & $0.88-1.13$ & $\theta .03$ \\
\hline Adjusted in per protocol population $\S$ & 0.95 & $0.84-1.09$ & $\theta .01$ & 1.00 & $0.88-1.14$ & 0.05 \\
\hline
\end{tabular}

CI denotes confidence interval, mRS modified Rankin Scale, OR odds ratio, smRSq simplified modified Rankin Scale questionnaire

*Noninferiority margin was 1.14 (i.e. an upper boundary of the $95 \% \mathrm{CI}$ for the $O \mathrm{R}$ with low-dose alteplase as compared with standard-dose alteplase of less than 1.14).

†ORs were estimated from logistic regression models. Each OR indicates the odds of death or disability (mRS 2 to 6 ). An OR greater than 1 favors standard-dose alteplase. Adjustment for site, time from stroke onset to randomisation, score as a continuous measure on the National Institutes of Health stroke scale (NIHSS), age, sex, ethnicity, pre-morbid score of 0 or 1 on the mRS, pre-morbid use of aspirin, other antiplatelet agent or warfarin, and any history of stroke, coronary artery disease, diabetes mellitus and atrial fibrillation.

FORs were estimated from ordinal logistic regression models. Each OR indicates the odds of an increase of 1 in the $m R S$ score. An OR greater than 1 favors standard-dose alteplase. Adjustment for same variables as in logistic regression models above.

§Per protocol population excluded patients who have one or more of the following protocol violations: age $<18$ years; final diagnosis not acute ischemic stroke; final diagnosis unknown/uncertain because of missing source documents or neuroimaging; baseline systolic bloød pressure $>185 \mathrm{mmHg}$; randomized $>4.5$ hours; failure to receive alteplase at either the correct bolus or infusion dose; failure to obtain a blind assessment of the 90 -day outcome. 


\section{Figures legend}

Figure 1. Bubble plot of agreement between smRSq and mRS at Day 90. Area of bubbles represent the count at each score.

Figure 2. ROC curves for predictive models of mRS and smRSq at Day 90. 
Figure 1. Agreement between smRSq and mRS at Day 90

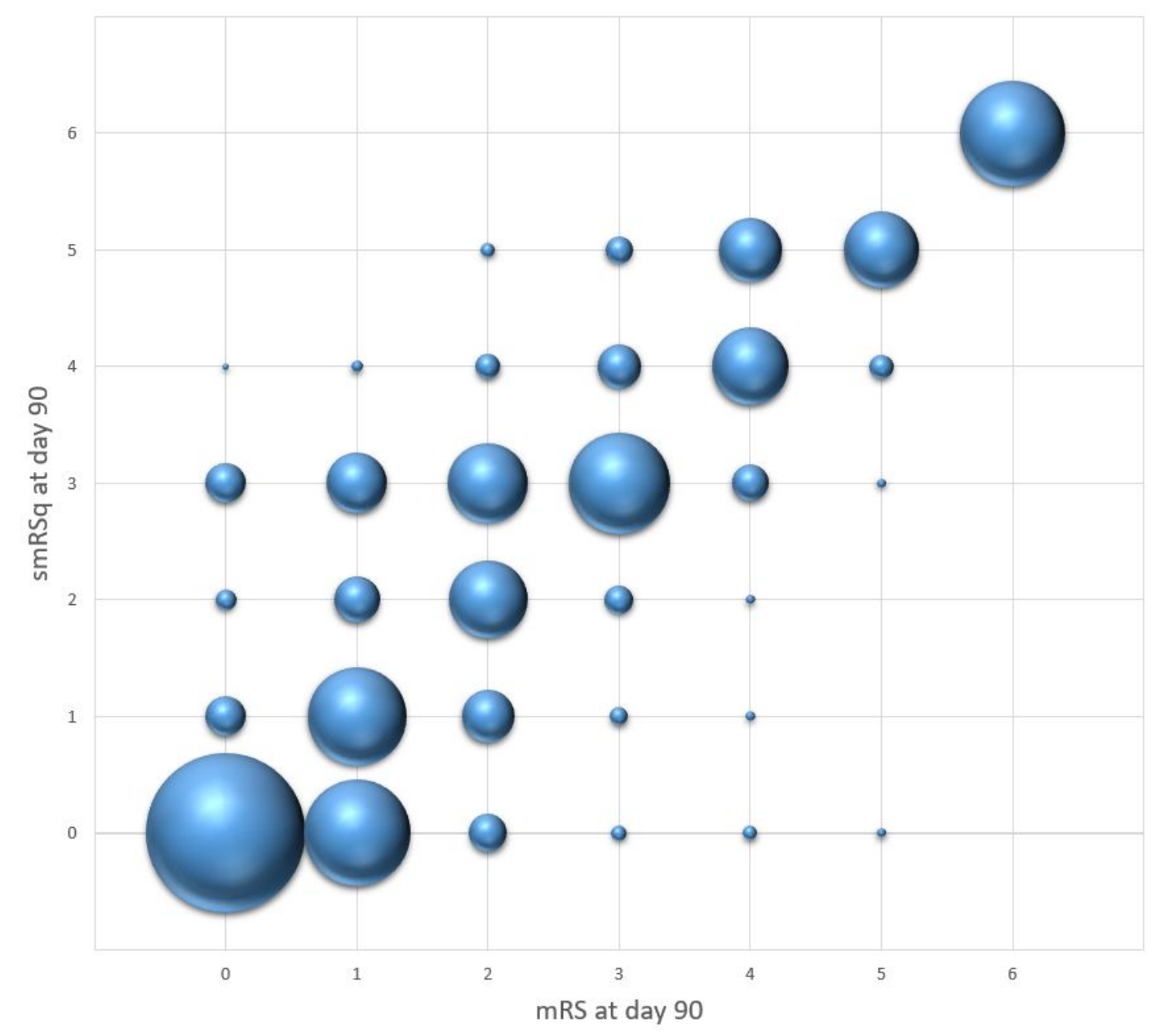


Page 23 of 22

International Journal of Stroke

Figure 2. ROC curves for the predictive models of $\mathrm{mRS}$ and smRSq at Day 90

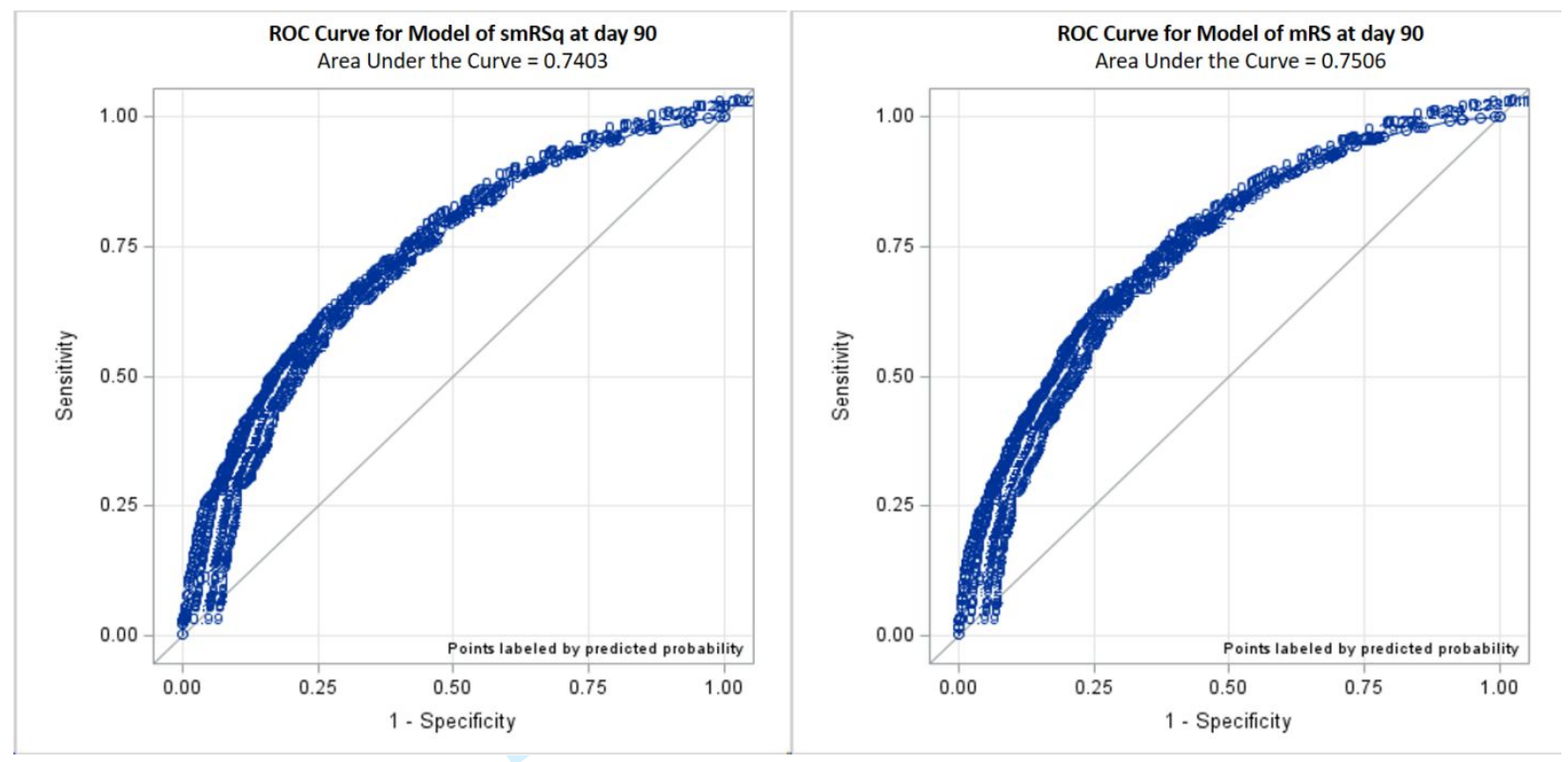

20 